\title{
New XY-Theta Positioning Table with Partially Decoupled Parallel Kinematics
}

\author{
Alexander $\mathrm{Yu}^{1}$, Ilian A. Bonev ${ }^{2}$, Paul Zsombor-Murray ${ }^{1}$ \\ ${ }^{1}$ Department of Mechanical Engineering, McGill University, Montreal, QC, Canada \\ ${ }^{2}$ Department of Automated Manufacturing Engineering, École de Technologie Supérieure, Montreal, QC, Canada \\ ilian.bonev@etsmtl.ca
}

\begin{abstract}
Parallel robots provide an efficient solution in a world demanding better positioning accuracy. These robots are preferred over their serial counterparts because each leg helps support the platform, thus reducing the need to add means for extra support. The problem with parallel robots though is that most are coupled and difficult to control. In this paper, new parallel robot architecture has been proposed that can deliver accurate movements in addition to being partially decoupled and fast. This is a significant technological advantage over current designs because a decoupled robot will be simpler to control as each actuator is independent. Furthermore, its displacement resolution is less variable throughout the workspace. To achieve the decoupled state, this parallel robot took the novel approach of having mixed legs. By comparing this robot to existing commercial devices through kinematic, singularity, workspace, velocity and dexterity analysis, this paper would like to show that this new design should be looked into further to create a precision positioning table that is parallel, decoupled, and highly accurate.
\end{abstract}

\section{INTRODUCTION}

The semiconductor industry and the MEMS field are both growing rapidly but according to the International Technology Roadmap for Semiconductors the manufacturing section is falling far behind in development. Issues such rapidity and platform accuracy are at the top of their list of problems that are to be solved for material handling systems, considering the fact that the next standard for wafer diameter will be $450 \mathrm{~mm}$. Increasing workspace and speed requirements makes most current position-table technology inadequate in the context of factory space shortage.

Various solutions have been proposed to the industry to increase positioning accuracy for lithography and micromanufacturing. Many companies sell commercial three-axis (XY $\theta$ ) positioning tables to address these needs (e.g., Newport, Rockwell Automation, Physik Instrumente, and Aerotech). Nearly all of them are based on serial kinematics. Namely, one linear stage moves along the $x$ axis, another, placed on top of the first, moves along the $y$ axis, while a rotary table mounted on top of the second linear stage allows for rotation about the $\mathrm{z}$ axis. This serial configuration has the advantage of having a simple control of motion. However, the first actuator has to support the weight of all other actuators. The device would have to be large enough, not only to support itself but to absorb any vibrations caused by the three motors. As a result, such a device would be both too sluggish and relatively big.

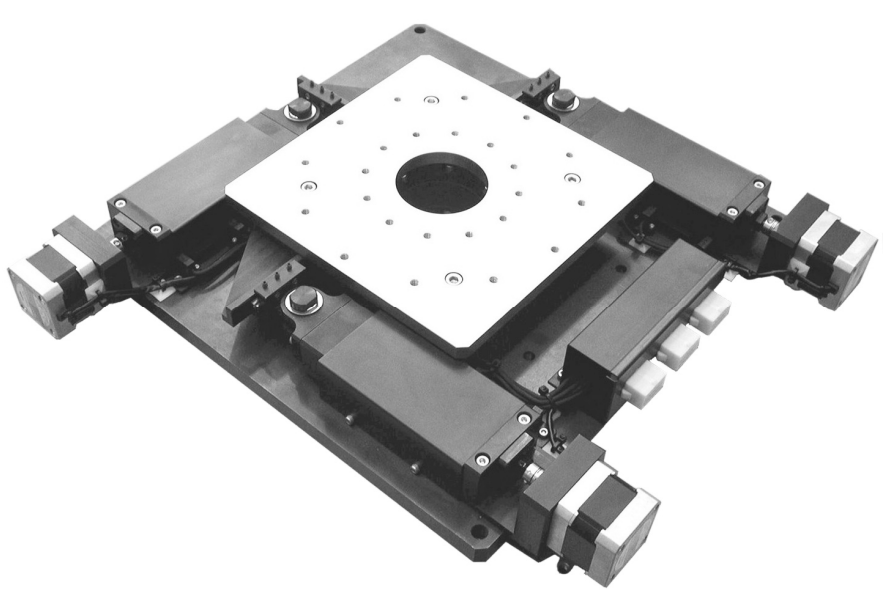

Fig. 1. NAF3 Alignment Stage (courtesy of Hephaist Seiko Co., Ltd.).

Research into the field of parallel structures is advantageous, because parallel robots are less bulky than serial ones. And since each leg is supporting the mobile platform, the robot can be considered stiffer than its serial counterparts. Therefore no extra structural support is needed to make the system rigid.

Hephaist Seiko, a company in Japan, has opted for a parallel robot solution for micro-positioning (Fig. 1). The company has made an innovative positioning table that utilizes a 3-PRP parallel architecture that allows all of its linear actuators to be base-mounted ( $P$ and $R$ stand for prismatic and revolute joints, respectively, and an underline indicates which joint is actuated). The resulting positioning table is very rigid since the mobile platform is directly supported by the actuators. However, this design has the problem of being highly coupled, meaning that to move in certain directions all three actuators must work in conjunction. Another recent adaptation of a 3-PRP planar parallel mechanism was suggested in [1], which uses a unique star-triangle setup for micro-positioning. This device is however as coupled as the Hephaist one.

A better solution to the accuracy problem would be a parallel robot that is decoupled or at least partially decoupled. The result will be a robot with the stiffness and rapidity of a parallel structure and the ease of control/programming that is associated with serial robots. Fully decoupled parallel robots were proposed in [2]. However, these parallel robots are not suitable for precision positioning devices because they have too many joints and offer little support to the mobile platform. 


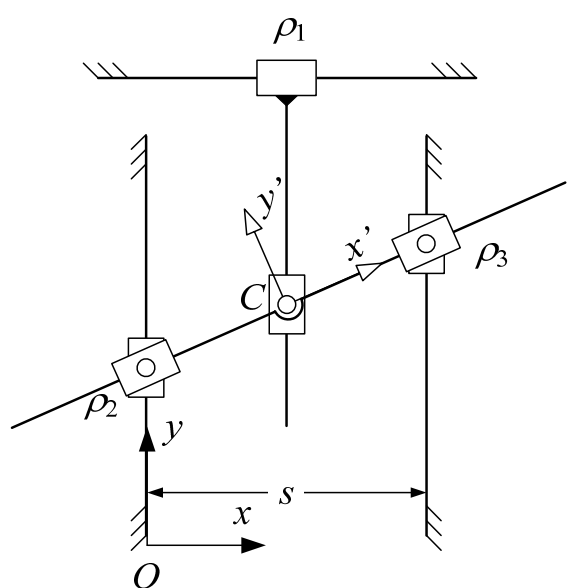

(a)

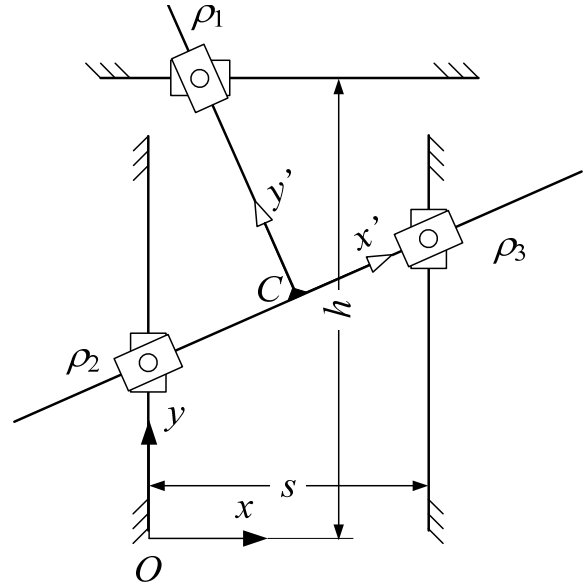

(b)

Fig.2. Schematics of both (a) the new parallel robot (patent pending) and (b) Hephaist's parallel robot.

This paper suggests a new architecture that is both partiallydecoupled and offers good support to the mobile platform. The paper will provide useful analysis on this new device, which will be shown in the next five sections. The following section will describe the architecture of the robot, including its mechanical design. The remainder will be devoted to kinematic, workspace, velocity and dexterity analysis of the new robot, with a comparison to the Hephaist design.

\section{NeW ARCHITECTURE}

The proposed architecture (patent pending) is a parallel robot with two $\underline{P} R P$ legs and one $\underline{P} P R$ leg (Fig. 2a). The directions of the actuators in the $\underline{P} R P$ leg are parallel to the $y$ axis, while the direction of the actuator in the $\underline{P} P R$ leg is parallel to the $x$ axis. The two passive prismatic joints on the mobile platform have parallel directions and the centers of the three revolute joints are collinear. The directions of the two prismatic joints in the $\underline{P P R}$ leg are normal. Consequently, if actuators 2 and 3 move in conjunction, at the same rate, the mobile platform uniquely translates along the $y$ axis. If the two move at opposite directions, a pure rotation about the $z$ axis could occur. Finally, actuator 1 directly controls the $x$ coordinate of the platform.

A physical design of this robot has being proposed for a first prototype and is shown in Fig. 3. It comprises three linear guides from LinTech (two from the 130 series and one from the 100 series). Each guide is screw-driven with a repeatability of $2.5 \mu \mathrm{m}$. The two LinTech 130 series linear guides are elevated on aluminum blocks to allow the LinTech 100 series linear guide to run below them. Each of the two 130 series linear guides will have a pivoting block attached onto the carriage. A steel shaft will be rigidly attached to one of the two pivoting blocks and, through a linear bearing, to the other pivoting block. The mobile platform will slide along this shaft through another pair of linear bearings. The carriage of an INA monorail guide will be attached to the carriage of the LinTech 100 series linear guide, so that the two guides are perpendicular. The monorail guide will be fixed to the tapered block, which holds a thrust bearing that is attached to the mobile platform.
Note that the LinTech 100 series linear guide has a wider carriage and a much higher loading capacity $(1400 \mathrm{~kg}$ compared to $90 \mathrm{~kg}$, for the 130 series), minimizing the deflection that would occur when the mobile platform is at its extreme position in $y$. Overall, this mechanical design provides high rigidity in all directions and particularly in the $z$ direction (normal to the plane of motion).

The motors that will drive the linear guides are three identical Cleveland Motion Controls BNL2310 servo motors, NEMA 23 size. Servo motors were chosen because they have excellent stiffness capabilities, allowing for quick starts and stops. These motors also have quick responses to control signals, making them ideal for this sort of application. No gear boxes will be used, since Acme precision ball screws of 0.2 in lead are used.

Finally, the 100 series and the two 130 series linear guides will have travel lengths of 8 in and 12 in, respectively. The distance $s$, as defined in Fig. 2a, is about 17 in. As a result, the mobile platform can rotate up to $\pm 35^{\circ}$ and translate inside a rectangle of 8 in by 12 in (when at $0^{\circ}$ ). The mobile platform is $6.5 \times 6.5$ in and the bottom plate is $20 \times 20$ in.

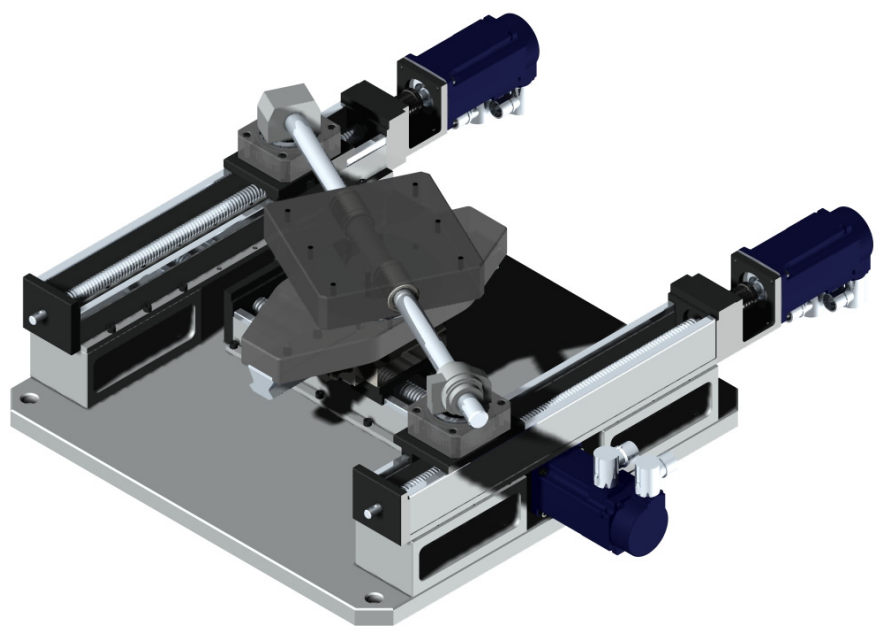

Fig. 3. 3D CAD model of the new parallel robot (patent pending). 


\section{DIRECT AND INVERSE KINEMATIC ANALYSIS}

Referring to Fig. $2 \mathrm{a}$ and $2 \mathrm{~b}$, a base reference frame $O x y$ is fixed at the base so that the center of the revolute joint of leg 2 lies on the $y$ axis, and a mobile reference frame $C x^{\prime} y^{\prime}$ is fixed to the mobile platform so that the centers of the revolute joints of legs 2 and 3 lie on the $x$ ' axis. In Fig. 2a, the origin $C$ coincides with the center of the revolute joint of leg 1 , while in Fig. 2, the origin $C$ is placed so that the center of the revolute joint of leg 1 lies on the $y$ ' axis. Finally, $\theta$ is the angle between the $x$ and $x$ ' axes.

Furthermore, $\rho_{1}$ is the active-joint variable defined as the distance between the $y$ axis and the center of the revolute joint of leg 1 , and $\rho_{2}$ and $\rho_{3}$ are the active-joint variables defined as the distances between the $x$ axis and the centers of the revolute joint of leg 2 and 3, respectively. In addition, $s$ is the horizontal distance between the centers of the revolute joints of legs 2 and 3. In Fig. 2b, only, $h$ is the distance between the center of the revolute joint of leg 3 and the $x$ axis.

\section{A. Hephaist's Parallel Robot}

Given the active-joint variables, we are able to uniquely define the position and orientation of the mobile platform. The orientation angle is easily obtained as

$$
\theta=\tan ^{-1}\left(\frac{\rho_{3}-\rho_{2}}{s}\right) .
$$

The position of the mobile platform is the intersection between the line connecting the centers of the revolute joints of legs 2 and 3, and the line connecting the center of the revolute joint of leg 1 and normal to the previous line. The resulting equations for $x$ and $y$ are:

$$
\begin{gathered}
x=\frac{s\left(\rho_{1} s+\left(h-\rho_{2}\right)\left(\rho_{3}-\rho_{2}\right)\right)}{s^{2}+\left(\rho_{3}-\rho_{2}\right)^{2}}, \\
y=\frac{s^{2} \rho_{2}+h\left(\rho_{3}-\rho_{2}\right)^{2}+s \rho_{1}\left(\rho_{3}-\rho_{2}\right)}{s^{2}+\left(\rho_{3}-\rho_{2}\right)^{2}} .
\end{gathered}
$$

The results appear to be rather complicated, despite having an easy process to solve for it. That is because the position of the mobile platform is dependant on all the active-joint variables, which shows that the robot has coupled input-output relationship.

The inverse kinematics is easier to solve for. Given the position and orientation of the mobile platform, the active-joint variables are obtained as

$$
\begin{gathered}
\rho_{1}=x-(h-y) \tan \theta \\
\rho_{2}=y-x \tan \theta \\
\rho_{3}=y+(s-x) \tan \theta
\end{gathered}
$$

Since (1-6) are always defined, assuming $s \neq 0$, it is evident that this parallel robot has no singularities. Note, that this is a great advantage over most parallel robots, which have intricate singularities.

\section{B. New Parallel Robot}

Given the active-joint variables, we are again able to uniquely define the position and orientation of the mobile platform. The orientation angle is defined by (1), while the position of the mobile platform is given by

$$
\begin{gathered}
x=\rho_{1} \\
y=\rho_{2}+\rho_{1}\left(\frac{\rho_{3}-\rho_{2}}{s}\right)
\end{gathered}
$$

As one can observe, the direct kinematic equations of the new parallel robot are much simpler than those for the Hephaist robot. Furthermore, $x$ is directly defined by actuator 1, thus the reason why our parallel robot is partially decoupled.

The inverse kinematic analysis is also simple. Given the position and orientation of the mobile platform, the active-joint variables are obtained as

$$
\begin{gathered}
\rho_{1}=x \\
\rho_{2}=y-x \tan \theta \\
\rho_{3}=y+(s-x) \tan \theta
\end{gathered}
$$

Similarly, it is evident that our parallel robot has no singularities, provided that $s$ is non-zero.

\section{WORKSPACE ANALYSIS}

The position workspace of both parallel robots is dependant on the orientation of the mobile platform. In both cases, the greater that orientation, the smaller the workspace area. To allow for fair comparison, we will assume that in both robots, the only mechanical limits restraining the workspace are the actuator travel lengths, which are the same:

$$
\rho_{1} \in\left[\rho_{1, \min }, \rho_{1, \max }\right], \rho_{2} \in\left[0, \rho_{2, \max }\right], \rho_{3} \in\left[0, \rho_{3, \max }\right],
$$

as shown in Fig. 4.

Under these conditions, the position workspace for both parallel robots can be easily obtained geometrically, as shown in Fig. 4, where the position workspace for a given orientation is the hatched region. In both robots, this workspace is delimited by two lines parallel to the $x$ ' axis and passing through the limits of actuators 1 and 2. In Hephaist's robot, this workspace is further delimited by two lines parallel to the $y$ ' axis and passing through the limits of actuator 1 . In our parallel robot, the position workspace is further delimited by two lines parallel to the $y$ axis and passing through the limits of actuator 1 .

From here, it is obvious that the position workspace of our parallel robot is greater than the position workspace of Hephaist's robot, for any non-zero orientation. Furthermore, the position workspace of our parallel robot is always centered between actuators 2 and 3, while the center of the position workspace of Hephaist's robot is hugely varying. This means that the region in which each point is accessible with at least one orientation in a given range, $\theta \in\left[-\theta_{\max }, \theta_{\max }\right]$, is much larger for our parallel robot. 


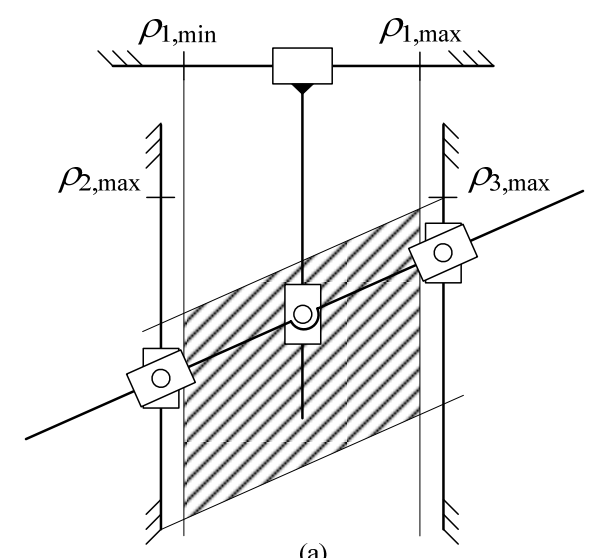

(a)

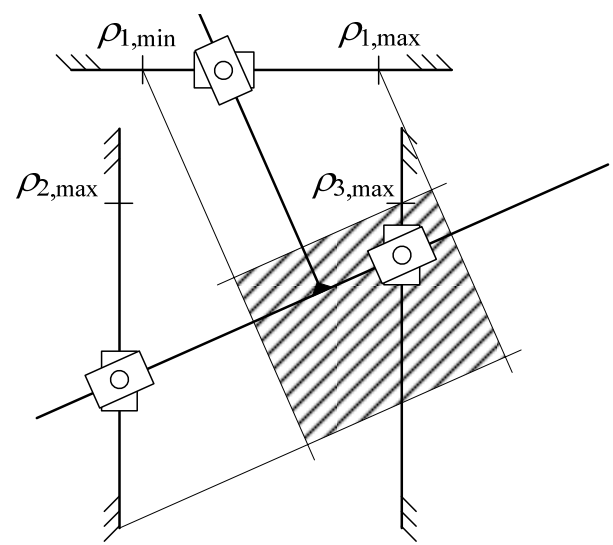

(b)

Fig.4. Position workspace for a given orientation of the mobile platform for both (a) the new parallel robot and (b) Hephaist's parallel robot.

\section{VELOCITY EQUATIONS AND JACOBIAN}

The velocity equations are essential for a precision positioning device, even if the velocity of the mobile platform is not needed. These equations define the Jacobian matrix which can be used to find useful information such as local and global dexterity. The Jacobian matrix is defined as follows:

$$
\dot{\rho}=\mathbf{J} \dot{\mathbf{q}} \text {, }
$$

where

$$
\dot{\boldsymbol{\rho}}=\left[\dot{\rho}_{1}, \dot{\rho}_{2}, \dot{\rho}_{3}\right]^{T}, \quad \dot{\mathbf{q}}=[\dot{x}, \dot{y}, \dot{\theta}]^{T} .
$$

By differentiating the inverse kinematic equations of Hephaist's robot (4-5), the following is obtained for its Jacobian:

$$
\mathbf{J}_{\text {Hephaist }}=\left[\begin{array}{ccc}
1 & \tan \theta & (y-h) \sec ^{2} \theta \\
-\tan \theta & 1 & -x \sec ^{2} \theta \\
-\tan \theta & 1 & (s-x) \sec ^{2} \theta
\end{array}\right] .
$$

Simiarly, by differentiating the inverse kinematic equations of our robot (9-11), the following is obtained for its Jacobian:

$$
\mathbf{J}_{\text {New }}=\left[\begin{array}{ccc}
1 & 0 & 0 \\
-\tan \theta & 1 & -x \sec ^{2} \theta \\
-\tan \theta & 1 & (s-x) \sec ^{2} \theta
\end{array}\right] .
$$

Note that the Jacobian of our robot is dependant on only $\theta$ and $x$. That is due to the fact the robot is partially-decoupled.

\section{LOCAL DEXTERITY ANALYSIS}

A dexterity analysis can be applied to the system now that the Jacobian matrix is known. Dexterity is defined as the capability of the robot to make accurate movements and is a measure of its kinematic accuracy [3]. To characterize the accuracy of a robot's dexterity a performance index was established based on the Jacobian matrix. The Jacobian matrix is used because it is the linear transformation between Cartesian and actuator velocities. The local dexterity is the quality of that transformation. Dexterity index will be defined as [3]:

$$
\xi=\frac{1}{\|\mathbf{J}\|\left\|\mathbf{J}^{-1}\right\|},
$$

where

$$
\|\mathbf{J}\|=\sqrt{\operatorname{tr}\left(\frac{1}{3} \mathbf{J J}^{T}\right)} .
$$

It is well known, that the dexterity index $\xi$ defined in the above way is not frame invariant since it depends on both position and orientation parameters. However, for the purposes of comparison, we can use this index if we use the same physical dimensions in both Hephaist's and our parallel robot. Namely, we use $s=h=10$ (in units that need not be specified) and present two typical dexterity plots in Figs. 5 and 6.
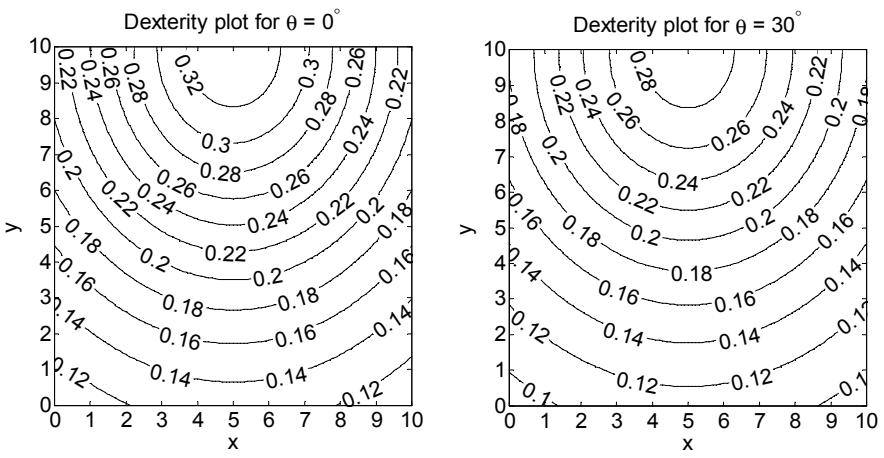

Fig. 5. Dexterity contour plots for Hephaist's robot for two orientations.
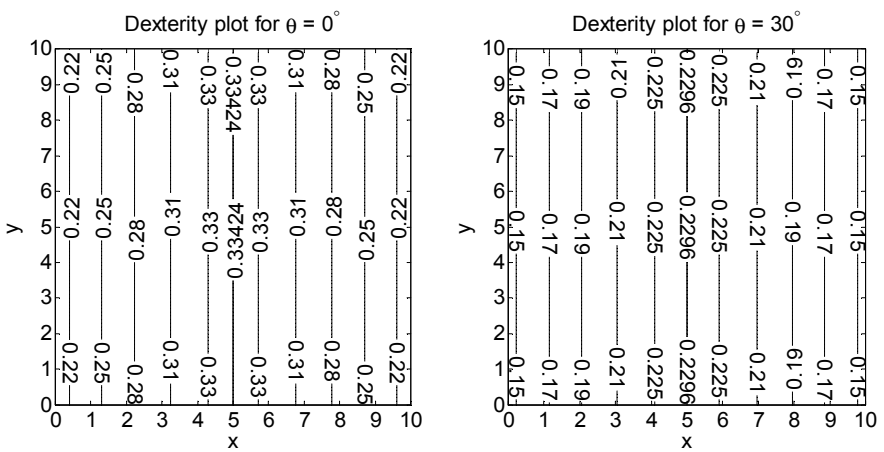

Fig. 6. Dexterity contour plots for our robot for the same two orientations. 
While the actual values for the local dexterity in each robot are irrelevant, two facts are obvious from Figs. 5 and 6 (and can be easily proven analytically). Firstly, the accuracy is reduced for both robots when the orientation angle increases. Secondly, the accuracy of Hephaist's parallel robot is proportional to the distance between the center of the mobile platform and the point $(s / 2, h)$. Yet, it is obvious from Fig. 1, that Hephaist's NAF3 alignment stage operates close to the point $(s / 2, h / 2)$, i.e., far from its maximum local dexterity. In contrast, the accuracy of our parallel robot is independent from the $y$ coordinate of the center of the mobile platform and is proportional to the distance between the center of the mobile platform and the line $x=s / 2$. Therefore, while the highest dexterity indices for both robots are comparable, our parallel robot actually achieves these values throughout the center of its workspace. In other words, it is obvious that our parallel robot is better than Hephaist's one in terms of kinematic accuracy.

\section{CONCLUSION}

In this paper, a new parallel architecture for precision positioning has been proposed. The new architecture is a modification of the architecture of an existing commercial alignment stage, but the actual mechanical design that was briefly discussed is completely different. Through kinematic analysis of both the existing and the new parallel robots, it was shown that the new one has simpler partially-decoupled kinematic equations, larger workspace, and better accuracy. Currently under construction, this device could be a better solution for accurate positioning for the semiconductor industry.

\section{ACKNOWLEDGMENTS}

The authors would like to thank Mr. Luc Bilodeau for creating the CAD model shown in Fig. 3, as well as to the Fonds québécois de la recherche sur la nature et les technologies who provided funding for building the first prototype.

\section{REFERENCES}

[1] S. Ronchi, O. Company, F. Pierrot, and A. Fournier, "PRP Planar Parallel Mechanism in Configurations Improving Displacement Resolution," Proceedings of the $1^{\text {st }}$ International Conference on Positioning Technology, Act-city, Hamamatsu, Japan, June 9-11, 2004.

[2] G. Grigore, "Fully-Isotropic Over-Constrained Planar Parallel Manipulators," Proceedings of 2004 IEEE/RSJ International Conference on Intelligent Robots and Systems, Sendai, Japan, September 28 - October 2, 2004.

[3] C. Gosselin, "The optimum design of robotic robots using dexterity indices," Robotics and Autonomous systems, Vol. 9, pp. 213-226, 1992.

[4] C. Gosselin "Stiffness Mapping for Parallel robots," IEEE Transactions on Robotics and Automation, Vol 6, No.3, pp. 377-382, 1990.

[5] C. Gosselin, S Lemieux and J.-P. Merlet, "A new architecture of planar three-degree-of-freedom parallel robot," Proceedings of the 1996 IEEE International Conference on Robotics and Automation, Minneapolis, Minnesota, USA, April, 1996.

[7] J. Angeles, Fundamentals of Robotic Mechanical System, Second Edition, Springer, 2003

[8] M.H.R. Daniali, P. Zsombor-Murray, and J. Angeles, "The Kinematics of 3-DOF Planar and Spherical Double-Triangular Parallel Robot," Computational Kinematics, J. Angeles et al. (eds.), Kluwer Academic Publishers, pp. 153-164, 1993.
[9] C. Gosselin and J. Angeles, "A Global Performance Index for the Kinematic Optimization of Robotic Robots," Journal of Mechanical Design, Vol. 113, No. 3, pp. 220-226, September 1991. 